\title{
BMJ
}

\section{Effectiveness of five different approaches in management of urinary tract infection: randomised controlled trial}

\author{
P Little, professor of primary care research, ${ }^{1} \mathrm{M}$ V Moore, senior lecturer, ${ }^{1} \mathrm{~S}$ Turner, trial manager, ${ }^{1} \mathrm{~K}$ Rumsby, \\ trial data coordinator, ${ }^{1} \mathrm{G}$ Warner, general practitioner, ${ }^{2} \mathrm{~J}$ A Lowes, consultant microbiologist, ${ }^{3} \mathrm{H}$ Smith, \\ professor of primary care, ${ }^{4} \mathrm{C}$ Hawke, public health physician, ${ }^{5} \mathrm{G}$ Leydon, senior research fellow, ${ }^{1} \mathrm{~A}$ Arscott, \\ research assistant, ${ }^{1} \mathrm{D}$ Turner, health economics research fellow, ${ }^{6} \mathrm{M}$ Mullee, senior lecturer in medical \\ statistics and director of $\mathrm{RDSU}^{7}$
}

${ }^{1}$ Primary Care Medical Group, Community Clinical Sciences Division, University of Southampton School of Medicine, Southampton S016 5ST

${ }^{2}$ Nightingale Surgery, Romsey, SO51 7QN

${ }^{3}$ Southampton Universities Hospital Trust Microbiology Laboratory, Southampton General Hospital, Southampton S016 6YD

${ }^{4}$ Brighton and Sussex Medica School, University of Sussex, Brighton BN1 9PX

${ }^{5}$ School of Rural Health, University of Sydney, Orange Campus,

PO Box 1191, Orange, NSW, Australia

${ }^{6}$ Wessex Institute, University of Southampton, Southampton

${ }^{7}$ Community Clinical Sciences Division (CCS), School of

Medicine, University of

Southampton, Public Health Sciences and Medical Statistics

Group, Southampton General Hospital, Southampton S016 6YD Correspondence to: P Little, University of Southampton, Aldermoor Health Centre, Southampton S016 5ST p.little@soton.ac.uk

Cite this as: BMJ 2010;340:c199 doi:10.1136/bmi.c199

\section{ABSTRACT}

Objective To assess the impact of different management strategies in urinary tract infections.

Design Randomised controlled trial.

Setting Primary care.

Participants 309 non-pregnant women aged 18-70 presenting with suspected urinary tract infection. Intervention Patients were randomised to five management approaches: empirical antibiotics; empirical delayed (by 48 hours) antibiotics; or targeted antibiotics based on a symptom score (two or more of urine cloudiness, urine smell, nocturia, or dysuria), a dipstick result (nitrite or both leucocytes and blood), or a positive result on midstream urine analysis. Self help advice was controlled in each group.

Main outcome measures Symptom severity (days 2 to 4) and duration, and use of antibiotics.

Results Patients had 3.5 days of moderately bad symptoms if they took antibiotics immediately. There were no significant differences in duration or severity of symptoms (mean frequency of symptoms on a 0 to 6 scale: immediate antibiotics 2.15, midstream urine 2.08, dipstick 1.74 , symptom score 1.77 , delayed antibiotics 2.11; likelihood ratio test for the five groups $\mathrm{P}=0.177$ ). There were differences in antibiotic use (immediate antibiotics $97 \%$, midstream urine $81 \%$, dipstick $80 \%$, symptom score $90 \%$, delayed antibiotics $77 \%$; $P=0.011$ ) and in sending midstream urine samples (immediate antibiotics $23 \%$, midstream urine $89 \%$, dipstick $36 \%$, symptom score $33 \%$, delayed antibiotics $15 \%$; P $<0.001$ ). Patients who waited at least 48 hours to start taking antibiotics reconsulted less (hazard ratio 0.57 (95\% confidence interval 0.36 to 0.89 ), $P=0.014$ ) but on average had symptoms for $37 \%$ longer than those taking immediate antibiotics (incident rate ratio 1.37 (1.11 to 1.68), $P=0.003)$, particularly the midstream urine group (73\% longer, $22 \%$ to $140 \%$; none of the other groups had more than $22 \%$ longer duration).

Conclusion All management strategies achieve similar symptom control. There is no advantage in routinely sending midstream urine samples for testing, and antibiotics targeted with dipstick tests with a delayed prescription as backup, or empirical delayed prescription, can help to reduce antibiotic use.

Study registration National Research Register N0484094184 ISRCTN: 03525333.

\section{INTRODUCTION}

Urinary tract infections are one of the most common conditions seen in female patients in general practice. ${ }^{1}$. These infections affect $50 \%$ of women at least once in their lives, ${ }^{2}$ and more than $20 \%$ of infections are resistant to trimethoprim and cephalosporins and 50\% to amoxicillin.

Urinary dipsticks are the most commonly used near patient test in primary care in the United Kingdom. The aim of using dipsticks is to target treatment to the $60 \%$ of women who have urinary tract infections, while minimising antibiotic use for women without infection. A previous validation study has shown that dipsticks and clinical scoring algorithms can potentially help improve the precision of diagnosis by improving the positive predictive values. ${ }^{3}$ If clinicians are to use dipsticks, however, they need to have strategies to deal with the poor negative predictive values. ${ }^{3}$ We know of no trial that has assessed dipstick or clinical management algorithms in comparison with the realistic alternatives. Previous studies have documented that delayed antibiotic prescribing in respiratory infections results in good symptom control, reduced belief in the effectiveness of antibiotics, and fewer repeat consultations. $^{4-6}$ We hypothesised that, compared with an immediate antibiotic prescription, other management strategies would result in worse symptom control, particularly in women asked to delay antibiotics empirically or while waiting for the result of midstream urine analysis.

We aimed to assess the effectiveness of management using dipstick or clinical algorithms compared with the alternative management strategies (empirical antibiotic treatment, delayed prescribing, and targeted prescribing based on midstream urine results). 


\section{METHODS}

Non-pregnant women with a suspected uncomplicated urinary tract infection (where antibiotic use was not mandatory) were recruited on presentation between June 2003 and September 2005 by 62 general practitioners and nurses in the south of England. Patients were excluded if immediate antibiotic treatment was necessary (if they were pregnant, if they had pyelonephritis, nausea, vomiting, or other severe systemic symptoms), if they were aged over 75 (in older people symptoms of urinary tract infection might be reported differently), or if they had psychosis or dementia or needed terminal care (as such patients might have been unable to complete the diary).

\section{Data collection}

The clinician documented baseline symptoms, clinical information, and demographic details (age, sex, and postcode). The patient kept a daily symptom diary, grading severity - 0 (no symptoms), 1 (a very slight problem), 2 (a slight problem), 3 (a moderately bad problem), 4 (a bad problem), 5 (a very bad problem), or 6 (as bad as it could be). The symptoms - dysuria, haematuria, frequency during day and night, "smelly urine," "tummy pain," generally feeling unwell, and restriction of daily activities - were based on the common presenting symptoms of urinary tract infection. ${ }^{7}$ The diary format was previously validated and shown to be sensitive to change for other acute infections. ${ }^{8}$ There were also questions (Likert scales) on belief in the effectiveness of antibiotics, which had good discriminant ability in previous studies, ${ }^{9}$ a self report questionnaire of medically unexplained somatic symptoms, ${ }^{10}$ and a list of other medical problems. To help improve completeness of the diaries, patients were phoned by the research assistant after three days to check there were no problems with the diary. No questions were asked about compliance or return to the surgery, since this could have altered patient behaviour. Completed diaries were returned to the surgery in a freepost envelope.

\section{Laboratory analysis}

Midstream urine samples were transported as in routine practice. European guidelines were used to document significant bacteriuria, which allows laboratory confirmation of diagnosis based on lower colony counts, ${ }^{11}$ similar to the latest UK standards www.hpa-standard methods.org.uk/documents/bsop/pdf/bsop41.pdf.

\section{Randomisation}

Patients were individually randomised within the consultation to one of five basic management groups: immediate antibiotics; delayed antibiotics; symptom score (antibiotics offered if two or more of urine cloudy on examination, urine offensive smell on examination, patient's report of moderately severe dysuria, or patient's report of nocturia); dipstick (antibiotics offered if nitrites or leucocytes and a trace of blood were detected); or midstream urine (symptomatic treatment until microbiology results available and then antibiotics targeted according to results) (see appendix on bmj.com). Randomisation was done with computer generated random number tables in blocks to provide reasonably balanced group numbers. Patients were allocated by the opening of a sealed numbered envelope containing the instruction sheets for one of the five management groups. Sealed envelopes were used to facilitate randomisation and to ensure that the sheets the clinician needed were immediately to hand. The potential to undermine randomisation was minimised by careful attention to maximising equipoise when presenting the study to clinicians, and by emphasising that women in all groups had access to antibiotics at their request. Sequential envelope use was also audited during the study to ensure integrity of randomisation.

Since normal management of urinary tract infection is to use immediate antibiotics, we judged that the control of self help advice in other groups was needed to avoid a major imbalance. Self help advice - regarding fluids, and the use of fruit juices, bicarbonate, and a self help leaflet - was controlled for by randomisation in a balanced factorial design (see appendix on bmj.com).

\section{Use of advice sheets}

For each patient, a structured advice sheet was used, which supported the initial management according to the proposed strategy - as used successfully in previous studies from this group. ${ }^{512-15}$ This study's pragmatic design allowed for variation according to negotiation with patients, as would happen in practice. ${ }^{5}$ Thus, although clinicians negotiated initial antibiotic management based on the sheets, they were allowed to provide immediate antibiotics in situations where there were strong patient expectations. Conversely, provided the initial proposed management was the management indicated by the sheet, doctors and nurses had discretion to do a dipstick test or order a midstream urine test-either negotiated because of patient pressure or expectation or because of clinical perceptions of the requirement for adequate documentation of diagnosis. Health professionals were asked to document what they did in each case, and we used this information in the analysis to assess whether the results were confounded by such behaviour. We did an in-depth review of 38 case notes with the health professionals concerned in the largest recruiting practice to assess why midstream urines and dipsticks were used when not indicated by the advice sheets.

A research assistant independent of the trial team and blinded to study group reviewed notes to document use of midstream urine analysis, antibiotic prescription, and referrals.

\section{Sample size calculation}

Calculation was done for $\alpha=0.05$ and $\beta=0.2$ with the NQuery sample size program for multiple groups. On the basis of previous consensus decisions, ${ }^{13}$ a small difference in symptoms was defined as, on 
Table 1|Baseline comparison of demographic and clinical characteristics of five main management groups. Figures are means (SD) unless specified

\begin{tabular}{|c|c|c|c|c|c|}
\hline & Immediate antibiotics & Midstream urine & Dipstick & Symptom & Delayed antibiotics \\
\hline $\begin{array}{l}\text { Frequency symptom severity } \\
\text { at point of randomisation* }\end{array}$ & $1.81(0.61)$ & $1.80(0.78)$ & $1.71(0.70)$ & $1.79(0.70)$ & $1.76(0.71)$ \\
\hline No (\%) married & $27 / 39(69)$ & $25 / 38(66)$ & $34 / 42(81)$ & $37 / 49(76)$ & $27 / 39(69)$ \\
\hline Age when left education (years) & $17.9(2.3)$ & $17.0(2.4)$ & $17.8(2.8)$ & $17.5(2.6)$ & $17.5(2.5)$ \\
\hline Age (years) & $43(16)$ & $41(16)$ & $43(15)$ & $45(15)$ & $39(16)$ \\
\hline No (IQR) of somatic symptoms† & $3(1-8)$ & $4(1-6)$ & $4(2-6)$ & $3(2-6)$ & $4(2-8)$ \\
\hline No (IQR) of medical problems $\ddagger$ & $2(1-8)$ & $2(1-6)$ & $2(1-6)$ & $3(1-6)$ & $2.5(1-8)$ \\
\hline No (\%) with previous cystitis & $40 / 46(87)$ & $35 / 41(85)$ & $32 / 39(82)$ & $43 / 50(86)$ & $35 / 41(85)$ \\
\hline
\end{tabular}

$\mathrm{IQR}=$ interquartile range.

*Mean of frequency symptoms at randomisation (daytime frequency, night frequency, dysuria); each symptom rated $0=$ no problem; $1=$ =mild problem, $2=$ moderately bad problem, $3=$ severe problem.

†On modified version of somatic symptom inventory (SSI). ${ }^{10}$ Patients indicate number of medically unexplained symptoms severe enough to interfere with normal life or which required seeing doctor.

fList of major medical problems (such as, back pain, diabetes, arthritis, etc, and free space for other) documented by patients and number listed counted.

average, one in two patients rating symptoms as a slight problem rather than a moderate problem (a difference of 0.5 on the diary score). Based on the means and standard deviation (SD) from the pilot study, assuming the midstream urine and delayed groups had diary scores 0.5 higher than those of the other groups in the three days after seeing the doctor, a sample size of 260 patients was required, allowing for up to $20 \%$ loss to follow-up.

We assessed the effect of management strategies using multiple regression on an intention to treat basis, mutually controlling for all interventions (Stata version 9, StataCorp, College Station, TX). No interaction between self help advice and the basic management strategies was found, so the main effects of the basic management strategies are presented controlling for self help advice. We used negative binomial regression for duration of symptoms (because of overdispersion), multiple linear regression for the severity of symptoms, logistic regression for antibiotic use and repeat consultations, and Cox regression for time to repeat consultation. Our primary assessment was of the overall significance of the main intervention factor using the likelihood ratio test. The estimates of most interest are the comparison of each group with the control group (immediate antibiotics, the most common management strategy used by clinicians). We therefore report the estimate of differences compared with the control group with their 95\% confidence intervals. We specified symptom severity as the primary outcome a priori. A previous factor analysis of an observational cohort study demonstrated two groups of symptoms: increased day frequency, increased night frequency, and urgency and dysuria ("frequency" symptoms; Cronbach's $\alpha=0.77)$; and abdominal pain, restricted activities, and feeling unwell ("unwell" symptoms; $\alpha=0.80$ ). The observational data suggested that the severity "frequency symptoms" would be most likely to differ with alternative strategies and thus the frequency score (presented as the mean score of all the above items) was specified as the primary outcome.
Since the study was individually randomised we did not control for practice or general practitioner in the models (and controlling for general practitioner did not alter the inferences).

\section{RESULTS}

Most general practitioners recruited few patients, citing lack of time as the major reason. Of those patients invited, most accepted, and there was no difference in characteristics of this patient group with those in other observational studies. ${ }^{3}$ There were no important differences by management group for the key baseline variables of age, symptom severity, past cystitis, somatic symptom inventory, and comorbidities (table 1). Although marital status did differ slightly by group, inclusion of this factor in the models did not confound the estimates.

There were differences between groups where clinicians reported sending a midstream urine sample to the laboratory at the index consultation (immediate antibiotics $23 \%(15 / 66)$, midstream urine $89 \%$ (48/54), dipstick 36\% (21/58), symptom score 33\% $(23 / 69)$, and delayed antibiotics $15 \%(9 / 62) ; \chi^{2}=81$; $\mathrm{P}<0.001)$. There were also differences in the number where dipstick results were documented (immediate antibiotics $50 \%$ (33/66), midstream urine 52\% (28/54), dipstick 95\% (55/58), symptom score 55\% $(38 / 69)$, delayed antibiotics $29 \%(18 / 62) ; \quad \chi^{2}=55$; $\mathrm{P}<0.001)$. Including these variables in the models (that is whether or not a midstream urine sample was sent or a dipstick result documented) did not alter the estimates of the main outcomes.

The detailed review and discussion of cases - where dipstick documentation and midstream urine analysis were not prompted by the advice sheets - confirmed that subversion of protocol had probably not occurred. The main reasons highlighted were: negotiation based on patient expectation, professional perception of the need for adequate documentation (dipstick results being regarded as useful even if management was not based on them), and occasionally clinical reasons (for 
Table 2 |Effect on symptom duration, symptom severity for the two to four days after seeing the health professional, antibiotic use, and reconsultation during follow-up*. Figures are means differences, incidence ratios, odds ratios, or hazard ratios with $95 \%$ confidence intervals

\begin{tabular}{|c|c|c|c|c|c|}
\hline & $\begin{array}{l}\text { Mean frequency symptom† } \\
\text { severity (mean difference) }\end{array}$ & $\begin{array}{l}\text { Duration of moderately bad } \\
\text { symptoms in days } \\
\text { (incidence ratio } \ddagger \text { ) }\end{array}$ & $\begin{array}{l}\text { Mean unwell symptom§ } \\
\text { severity (mean difference) }\end{array}$ & $\begin{array}{c}\text { No (\%) who used antibiotics } \\
\text { odds ratio) }\end{array}$ & $\begin{array}{l}\text { Time to reconsultation } \\
\text { (hazard ratio) } \dagger\end{array}$ \\
\hline Immediate antibiotics & 2.15 (SD 1.18) & 1 & 1.60 (SD 1.30) & $58 / 60$ (97\%) & 1 \\
\hline Midstream urine & $2.08(-0.07 ;-0.51$ to 0.37$)$ & $1.21(0.92$ to 1.61$)$ & $1.66(0.05 ;-0.44$ to 0.55$)$ & $81 \%(38 / 47), 0.15$ (0.03 to 0.73$)$ & 0.81 (0.47 to 1.39$)$ \\
\hline Dipstick & $1.74(-0.40 ;-0.85$ to 0.04$)$ & 0.91 (0.68 to 1.22$)$ & $1.32(-0.28 ;-0.77$ to 0.20$)$ & $40 / 50(80 \%), 0.13$ (0.03 to 0.63$)$ & $0.98(0.58$ to 1.65$)$ \\
\hline Symptom score & $1.77(-0.38 ;-0.79$ to 0.04$)$ & $1.11(0.85$ to 1.44$)$ & $1.26(-0.35 ;-0.80$ to 0.11$)$ & $52 / 58(90 \%), 0.29$ (0.06 to 1.55$)$ & $0.73(0.43$ to 1.22$)$ \\
\hline Delayed antibiotics & $2.11(-0.04 ;-0.47$ to 0.40$)$ & $1.12(0.85$ to 1.47$)$ & $1.43(-0.18 ;-0.65$ to 0.30$)$ & 41/53 (77\%), 0.12 (0.03 to 0.59) & $0.60(0.35$ to 1.05$)$ \\
\hline $\mathrm{P}$ (likelihood ratio test) & 0.177 & 0.369 & 0.392 & 0.011 & 0.345 \\
\hline \multicolumn{6}{|c|}{$\begin{array}{l}\text { *All estimates are adjusted for self help advice given. Frequency symptoms are also adjusted for the estimate of severity of frequency symptoms at baseline. } \\
\text { Where differences are quoted, these are estimated differences compared with immediate antibiotics. Thus taking first row midstream urine group has mean frequency symptoms } 0.07 \text { lower } \\
\text { than immediate group, incidence rate ratio of } 1.21 \text { compared with immediate group (that is, } 21 \% \text { longer duration of symptoms), and unwell symptoms } 0.05 \text { higher than immediate group. } \\
\text { †Mean score for four items: increased day frequency, increased night frequency, urgency, and dysuria. Symptom severity: } 0=\text { no problem, } 6=a s \text { bad as it could be. } \\
\text { fFor illustration, estimated duration of symptoms rated moderately bad or worse in groups based on mean of } 3.54 \text { days in immediate group and with incidence rate ratios, are } 4.28 \text { days fo } \\
\text { midstream urine, } 3.22 \text { days for dipstick tests, } 3.93 \text { days for symptom score, and } 3.96 \text { days for delayed antibiotics. } \\
\text { §Mean score for three items: abdominal pain, restricted activities, and feeling unwell. Symptom severity: } 0=\text { no problem, } 6=\text { as bad as it could be. }\end{array}$} \\
\hline
\end{tabular}

example, a higher risk of complication was expected; a more definite initial diagnosis was required) were cited for unprompted investigations.

\section{Symptoms}

The average duration of symptoms rated as moderately bad or worse with immediate antibiotics was 3 . 5 days. Overall, there were no significant differences in symptom duration, severity of frequency symptoms, or severity of unwell symptoms between the antibiotic management strategies (table 2). The confidence intervals for symptom severity were all, with the exception of the midstream urine group, within the limit of SD 0.5 - the clinically important difference we specified a priori. The confidence intervals here are particularly helpful in showing that the estimates for the dipstick, symptom, and delayed antibiotics groups are unlikely to be 0.5 worse on the diary score - that is, unlikely to do harm - which is what clinicians need to know if they are considering changing from their current empirical treatment strategy. Those women who delayed antibiotics for 48 hours or more, however, were likely to have $37 \%$ longer duration of symptoms rated as moderately bad (incidence rate ratio 1.37 (95\% confidence interval 1.11 to 1.68$), \mathrm{P}<0.001$ ). The effect of delaying more than 48 hours predominantly applied to the midstream urine group (likelihood ratio test for interaction for the five groups $\mathrm{P}=0.08$; for midstream urine group $v$ the other groups $\mathrm{P}=0.02$ ) (table 3 ). Women delayed longest in both the midstream urine and the delayed groups (the average day starting antibiotics was 1.19 for immediate antibiotics, 2.18 for midstream urine, 1.43 for dipstick testing, 1.40 for symptom score, and 2.21 for delayed antibiotics).

\section{Use of antibiotics}

Of women in the midstream urine group, 66\% (36/54) had a confirmed urinary tract infection. There were significant differences in the number of women who waited at least 48 hours before taking antibiotics (immediate antibiotics 8\% (5/60); midstream urine
43\% (20/47); dipstick 30\% (15/50), symptom score $19 \%(11 / 58)$, and delayed antibiotics 53\% (28/53); $\chi^{2}$ $34 ; \mathrm{P}<0.001)$; also in the number taking antibiotics (immediate antibiotics $97 \%$ (58/60), midstream urine $81 \%$ (38/47), dipstick 80\% (40/50), symptom score $90 \%$ (52/58), delayed antibiotics 77\% (41/53); $\chi^{2} 11.7$; $\mathrm{P}=0.02)$. Women had similar beliefs in the effectiveness of antibiotics (immediate antibiotics 72\% (44/61), midstream urine 74\% (34/46), dipstick 79\% (37/47), symptom score $73 \%(41 / 56)$, delayed antibiotics $72 \%(36 / 50))$.

\section{Use of resources}

There was little difference between groups for further contacts recorded in the four weeks after consent (immediate antibiotics 6/58 (10\%), midstream urine 9/52 (17\%), dipstick 6/51 (12\%) symptom score 8/64 $(13 \%)$, delayed antibiotics $5 / 58(9 \%) ; \mathrm{P}=0.79)$, nor use of midstream urine analysis over the next month (immediate antibiotics 3/58 (5\%), midstream urine $3 / 52(6 \%)$, dipstick $4 / 51(8 \%)$, symptom score $5 / 64$ $(8 \%)$, delayed antibiotics $3 / 58(5 \%) \mathrm{P}=0.95)$. The average follow-up was 575 days (range 35 to 968). During follow-up there was no overall difference in time to reconsultation, but there was non-significant evidence that repeat consultations might be reduced in the delayed group (table 2). ${ }^{6}$ As some data for time to first reconsultations were missing for Cox regression, we also used more complete data to assess whether reconsultation had occurred, controlling for time between randomisation and notes review: in the immediate antibiotics group 32/58 (55\%) returned, and other groups reconsulted less: midstream urine odds ratio 0.65 ( 0.30 to 1.40$)$; dipstick 0.87 ( 0.40 to $1.90)$; symptom score 0.57 (0.27 to 1.18 ); and delayed 0.44 (0.21 to 0.95$)$. Patients who waited for at least 48 hours before using their prescription reconsulted less (hazard ratio 0.57 (0.36 to 0.89 ); $\mathrm{P}=0.014$ ). No major adverse events (major illness, admission to hospital, death) were reported for any group, and no significant differences in skin rash or thrush were reported. 
Table 3 | Estimates of symptom duration ( $95 \%$ confidence interval) for women who delayed antibiotics by 48 hours or more

\begin{tabular}{lcc} 
Immediate antibiotics & $\begin{array}{c}\text { Duration of moderately bad symptoms in days } \\
\text { (negative binomial; incidence rate ratio) } \\
\text { Incidence rate ratio set to } 1.00\end{array}$ & $\begin{array}{c}\text { Net effect of delaying } \\
\text { in each group* }\end{array}$ \\
\hline Midstream urine & $0.82(0.84$ to 2.83$)$ & $1.73(1.22$ to 2.44$)$ \\
\hline Dipstick & $0.84(0.61$ to 1.17$)$ & $1.20(0.78$ to 1.85$)$ \\
\hline Symptom score & $1.13(0.85$ to 1.50$)$ & $0.96(0.59$ to 1.57$)$ \\
\hline Delayed & $1.06(0.74$ to 1.51$)$ & - \\
\hline Taken on third day or later & $1.54(0.84$ to 2.83$)$ & - \\
\hline Interaction terms & & - \\
\hline Midstream urine/antibiotics taken on third day or later & $1.37(0.66$ to 2.85$)$ & - \\
\hline Dipstick/antibiotics taken on third day or later & $0.92(0.43$ to 1.99$)$ & - \\
\hline Symptom score/antibiotics taken on third day or later & $0.55(0.25$ to 1.20$)$ & - \\
\hline Delayed/antibiotics taken on third day or later & $0.74(0.36$ to 1.53$)$ & - \\
\hline
\end{tabular}

*Net effect=effect in group xeffect of taking after 3 days×interaction term for that group-for example, effect of delaying for more than 48 hours in midstream urine group $=0.82 \times 1.54 \times 1.37=1.73$.

\section{DISCUSSION}

All five management strategies for managing urinary tract infection in primary care achieved similar symptom control, and we found no advantage in routinely sending midstream urine samples for laboratory testing. Antibiotic use targeted with dipstick testing with a delayed prescription as backup or empirical delayed prescription helped to reduce antibiotic use.

\section{Potential limitations}

Patients' diary records for acute illness are likely to be reliable, and supporting each strategy in a structured manner minimises any differential placebo effects. ${ }^{5}$ Type I error is possible for the subgroup analyses (which must be interpreted with caution), and type II error is unlikely (we had complete results in $277(90 \%)$ patients so had more power than originally calculated). There was no evidence from auditing the use of envelopes, nor from the baseline tables, that randomisation was subverted. When there were modest differences between advice groups, we tested for confounding and found no evidence. In particular, although frequency symptoms were slightly (though not significantly) different at baseline between groups, controlling for baseline severity did not alter the inferences. The response to invitation, the mixed locations of practices, the range of demographics, and the similarity to observational cohorts ${ }^{3}$ should make these results generalisable. There was group differentiation in dipstick use, midstream urine analysis, and the willingness of women to delay the use of antibiotics, but a detailed review of cases suggested that clinicians were likely to want to do a midstream urine test or document dipstick results in a few patients irrespective of initial antibiotic policy-sometimes because of patients' expectation (which might be expected to change over time as doctors' behaviour changes), ${ }^{6}$ sometimes for legitimate clinical reasons (such as uncertainty about development of complications), and occasionally because of an overly optimistic view of the accuracy of dipsticks (such as wanting adequate documentation, which might be misguided given the poor negative predictive values of dipsticks). ${ }^{3}$ Estimates of effect were little altered when these behaviours (that is, ordering midstream urine analysis or dipstick documentation) were included in the model, which suggests there was little confounding of results by such behaviours.

\section{Symptom control}

The upper confidence intervals for the main outcome (frequency symptoms) suggest that alternative strategies are unlikely to result in poor symptom control (around 18\% worsening for the midstream urine or delayed groups, and $2 \%$ in dipstick and symptom score groups). There was some evidence that if women waited more than 48 hour they reconsulted less but had poorer control of symptoms, particularly for the midstream urine group. The finding of worse symptoms in such patients is in agreement with evidence of the effect of antibiotics from observational studies and trials. ${ }^{1617}$ Why the delayed group who waited a similar time did not have increased symptoms, however, is unclear. This might be a chance finding, but it might be because women in the midstream urine group find passively waiting for a laboratory result to be more distressing than being given the freedom to choose when to stop the delay according to their symptoms (the empirical delayed group). A previous study treated women negative for nitrite and leucocyte esterase with antibiotics and found symptomatic benefit, ${ }^{18}$ but as about a third of women who have negative results for all important dipstick tests (that is, not only nitrite and leucocyte but also blood) still have urinary tract infection based on rigorous laboratory confirmation, ${ }^{3}$ the finding of a benefit in women with negative dipstick results ${ }^{18}$ might be expected. The poor negative predictive value of dipsticks suggests that the offer of some kind of antibiotic safety net - such as delayed prescribing - to women with negative dipstick results is reasonable.

\section{Antibiotic use}

A modest reduction in antibiotic use (20-25\%) was achieved in all groups except the symptom score group. While these reductions are probably useful for public health, ${ }^{19}$ and the effect might plausibly increase 


\section{WHAT IS ALREADY KNOWN ON THIS TOPIC}

Urinary tract infection is distressing, and although antibiotics probably help symptoms, an immediate antibiotic prescription might not always be necessary

We know of no trials comparing the commonly used management strategies of empirical delayed prescribing, targeting by dipstick, targeting by symptom pattern, or waiting for the midstream urine result

\section{WHAT THIS STUDY ADDS}

\section{All management strategies achieve similar symptom control}

There is no advantage to routinely sending midstream urine samples for testing

Antibiotics targeted with dipstick tests with a delayed prescription as backup, or empirical delayed prescription, can help reduce antibiotic use

with time as patient expectations change,${ }^{6}$ the magnitude of the effect is in contrast to delayed antibiotic prescription among patients with respiratory infections. ${ }^{513}$ This is perhaps not surprising given that few respiratory infections are bacterial whereas most suspected urinary tract infections are $(66 \%$ of the midstream urine group had confirmed infection, similar to the previous study). ${ }^{3}$ There was some evidence that delayed prescribing might reduce reconsultation as we hypothesised, ${ }^{6}$ but this was not significant, probably because of the relatively low power of this analysis.

\section{Use of midstream urine analysis}

There was no evidence that either using midstream urine analysis as an initial strategy to guide antibiotic prescribing or the use of midstream urine samples by doctors as part of their overall clinical management made any difference to ordering analysis during follow-up or other outcomes. As with antibiotic prescribing, as perceptions change among patients and doctors regarding the need for midstream urine analysis in clinical management of uncomplicated infections, use of laboratory resources for such an unnecessary investigation could be significantly reduced.

\section{Conclusion}

All management strategies achieve similar symptom control. There is no advantage in routinely sending midstream urine samples, and patients who delay waiting for results of such analysis by more than 48 hours might have poor symptom control. Antibiotics targeted with dipstick tests with a delayed prescription as backup, or empirical delayed prescription help reduce antibiotic use.

We are grateful for the time given by patients and clinicians. We thank Bayer for provision of the 8SG strips.

Contributors: PL and MVM had the original ideas for the study. All authors developed the protocol. ST ran the study on a day to day basis, KR managed the database. PL and MM performed the analysis: $P L, M V M$, and MM are guarantors. All authors contributed to writing the paper Funding: This study was funded by the Health Technology Programme of UK NHS Research and Development. The researchers are independent of the funders, and the funders were not involved in management or analysis of the project.

Competing interests: JAL has been paid to attend consultancy workshops by Bayer and is currently working in collaboration with Bayer in unpaid capacity.

Ethical approval: This study was approved by the South West MReC ethics committee and informed consent was given by all patients. Data sharing: No additional data available; the authors are willing to share data where appropriate-for example, for use in individual patient data meta-analysis

1 HMSO, OPCS. Morbidity statistics from general practice: fourth national study 1991. 1st ed. HMSO, 1994

2 Foxman B. Epidemiology of urinary tract infections: incidence, morbidity, and economic costs. Dis Mon 2003;49:53-70.

3 Little P, Turner S, Rumsby K, Warner G, Moore M, Lowes A, et al. Developing clinical rules to predict urinary tract infection in primary care settings: sensitivity and specificity of near patient tests (dipsticks) and clinical scores. Br J Gen Pract 2006;529:606-12.

4 Little P, Gould C, Williamson I, Warner G, Gantley M, Kinmonth A. Clinical and psychological predictors of illness duration from randomised controlled trial of prescribing strategies for sore throat. BMJ 1999;319:736-7.

5 Little PS, Williamson I, Warner G, Gould C, Gantley M, Kinmonth AL. An open randomised trial of prescribing strategies for sore throat. BMJ 1997;314:722-7.

6 Little PS, Gould C, Williamson.I., Warner G, Gantley M, Kinmonth AL. Reattendance and complications in a randomised trial of prescribing strategies for sore throat: the medicalising effect of prescribing antibiotics. BMJ 1997;315:350-2.

7 Dobbs FF, Fleming DM. A simple scoring system for evaluating symptoms, history and urine dipstick testing in the diagnosis of urinary tract infection. J R Coll Gen Pract 1987;37:100-4.

8 Watson L, Little P, Williamson I, Moore M, Warner G. Validation study of a diary for use in acute lower respiratory tract infection. Fam Pract 2001;18:553-554.

9 Little PS, Williamson I, Warner G, Gould C, Gantley M, Kinmonth AL. An open randomised trial of prescribing strategies for sore throat. BMJ 1997;314:722-7.

10 Peveler R, Kilkenny L, Kinmonth A. Medically unexplained physical symptoms in primary care: a comparison of self report screening questionnaires and clinical opinion. J Psychosom Res 1997;42:245-52.

11 European Confederation of Laboratory Medicine. ECLM-European Urinanalysis Group European analysis guidelines. Scand J Clin Lab Invest 2000;60:1-96.

12 Little P, Griffin S, Kelly J, Dickson N, Sadler C. Effect of educational leaflets and questions on knowledge of contraception in women taking the combined oral contraceptive pill: randomised controlled trial. BMJ 1998;316:1948-52.

13 Little P, Rumsby K, Kelly J, Watson L, Moore M, Warner G, et al. Information leaflet and antibiotic prescribing strategies for acute lower respiratory tract infection: a randomised controlled trial. JAMA 2005;293:3029-35.

14 Little P, Gould C, Williamson I, Moore M, Warner G, Dunleavey J. A pragmatic randomised controlled trial of two prescribing strategies for acute otitis media. BMJ 2001;322:336-42.

15 Little P, Kelly J, Barnett J, Dorward M, Warm D, Margetts B. Randomised controlled factorial trial of dietary advice for patients with a single high blood pressure reading in primary care. $B M$ J 2004;328:1054-60.

16 Christiaens T, DeMeyere M, Verschraegen G, Peersman W, Heytens S, De Maeseneer J. Randomised controlled trial of nitrofurantoin versus placebo in the treatment of uncomplicated urinary tract infection in adult women. BrJ Gen Pract 2002;52:729-34.

17 Little P, Merriman R, Turner S, Rumsby K, Warner G, Lowes JA, et al. Presentation, pattern, and natural course of severe symptoms, and role of antibiotics and antibiotic resistance among patients presenting with suspected uncomplicated urinary tract infection in primary care: observational study. $B M$ J 2010;doi:10.1136/bmj.b5633.

18 Richards D, Toop L, Chambers S, Fletcher L. Response to antibiotics of women with symptoms of urinary tract infection but negative dipstick urine test results: double blind randomised controlled trial. BMJ 2005;331;143.

19 House of Lords Select Committee on Science and Technology: 7th report. Occasional Report 1998. 